\title{
Cambios en la cobertura de manglares en Bahía Culebra, Pacífico Norte de Costa Rica (1945-2010)
}

\author{
Catalina Benavides-Varela ${ }^{1,2}$, Jimena Samper-Villarreal $^{1} \&$ Jorge Cortés $^{1,2}$ \\ 1. Centro de Investigación en Ciencias del Mar y Limnología (CIMAR), Ciudad de la Investigación, Universidad de \\ Costa Rica, San Pedro, 11501-2060 San José, Costa Rica; tabebuiaguayacan@gmail.com; \\ jimena.samper@gmail.com; jorge.cortes@ucr.ac.cr \\ 2. Escuela de Biología, Universidad de Costa Rica, San Pedro, 11501-2060 San José, Costa Rica.
}

$$
\text { Recibido 15-X-2015. Corregido 18-III-2016. } \quad \text { Aceptado 14-IV-2016. }
$$

\begin{abstract}
Changes in mangrove coverage in Culebra Bay, North Pacific of Costa Rica (1945-2010). Despite the economic and environmental services that mangroves provide, they continue to be threatened by overexploitation, pollution, and land use change. Costa Rica has mangrove areas on the Pacific and Caribbean coasts, and cover has been declining since the 1980s. However, data on mangrove coverage are not continually updated and are often based on inaccurate estimates. It is therefore necessary to assess the current extension and variation of the mangrove cover in recent years, to determine changes. The mangrove cover was analyzed in two mangrove forests located in Bahía Culebra, North Pacific: Iguanita and Playa Panamá. For this, aerial photographs and satellite imagery were used to study changes for a 65 year period (1945-2010). Spatio-temporal changes were found in mangrove coverage, adjacent forests and areas without vegetation. Lower mangrove cover occurred during the 1970s (28.4 ha in Iguanita and 4.8 ha in Playa Panamá); but increased in recent years (38.9 ha in Iguanita and 12.0 ha in Panamá). Changes in forest cover by the Iguanita and Playa Panama mangroves were related to the history of land use around Bahía Culebra. Before 1980, there was extensive and intensive cattle ranching, increasing the deforestation rate; after that year, these practices were abandoned and secondary forest coverage increased until 2000. To ensure the adequate protection of mangroves, it is not only important to protect mangrove forests, but it is also necessary to establish buffer zones on their surroundings, to mitigate and/or reduce possible impacts. Rev. Biol. Trop. 64 (3): 955-964. Epub 2016 September 01.
\end{abstract}

Key words: aerial photographs, Central America, coastal environments, cover change, mangroves, GIS, satellite imagery.

Los manglares son ecosistemas reconocidos por su importancia ecológica y económica, su riqueza natural y los servicios ambientales que prestan (Ewel, Twilley, \& Ong, 1998; Duke et al., 2007). Sin embargo, son amenazados por la sobreexplotación, la contaminación y el cambio de uso de suelo (FAO, 2007a; Polidoro et al., 2010). La agricultura, la acuacultura, la extracción de sal y la expansión urbana, especialmente ligada al turismo, reducen la cobertura de manglar (Soto, 1988; Pizarro \& Angulo, 1993; Bilio, Coze, \& Portocarrero, 1999; FAO, 2007a; Flores-Mejía, Aguirre-Vallejo, FloresHernández, \& Guarddo-Govea, 2010; Polidoro et al., 2010; Vargas, 2010; Samper-Villarreal Cortés, \& Benavides-Varela, 2012). Además de esto, modelos de predicción sobre las consecuencias del cambio climático indican que habrá un fuerte impacto en los ecosistemas costeros (Alongi, 2008). Todos estos factores hacen de los manglares uno de los ecosistemas más amenazados del mundo (Duke et al., 2007; Polidoro et al., 2010).

Costa Rica tiene importantes áreas de manglar en las costas pacífica y caribeña. La mayor parte $(99 \%)$ se encuentra en la costa pacífica, principalmente en el Golfo de Nicoya y en el Golfo Dulce (Zamora-Trejos, 2006). 
Según estimaciones de la FAO (2007b), en Costa Rica la cobertura de manglar disminuyó de 63400 hectáreas en 1980 a 41000 hectáreas en 2005 (Pizarro \& Angulo, 1993). MIRENEM (1993) registró una pérdida de un $27 \%$ en la cobertura de manglar del país entre 1964 y 1989. Sin embargo, estos datos no están actualizados y están basados en estimaciones poco precisas, por lo que no hay una valoración exacta de cuál es la extensión actual de los manglares en Costa Rica, ni de cuál ha sido la tasa de cambio de la cobertura en los últimos años. Esta información es necesaria para poder determinar cambios en la cobertura a futuro. Se sabe que muchos de los otros manglares han sido deteriorados (Morales-Cabalceta, 1983; Soto, 1988) y algunos no han sido descritos (Zamora-Trejos \& Cortés, 2009). Es probable que se haya perdido cobertura, a pesar de que están protegidos.

Los manglares del Pacífico Norte de Costa Rica, en comparación con otros manglares del país, se caracterizan por ser de menor extensión, tener un menor número de especies de mangle y la vegetación, por lo general, es más pequeña (Soto \& Jiménez, 1982; Jiménez \& Soto, 1985; Jiménez, 1994; Zamora-Trejos \& Cortés, 2009; Loría-Naranjo, Samper-Villarreal, \& Cortés, 2014). Dado que los manglares de Bahía Culebra están inmersos entre complejos turísticos, es necesario conocer si ha habido cambios en su cobertura.

El objetivo de esta investigación fue estudiar la cobertura de dos manglares a lo largo del tiempo: Iguanita y Playa Panamá, ubicados en la región de Bahía Culebra, Pacífico Norte de Costa Rica. Para esto se analizaron los cambios durante un período de 65 años utilizando fotografías aéreas e imágenes satelitales; y se determinó su extensión más reciente como una referencia para el futuro.

\section{MATERIALES Y MÉTODOS}

Sitio de estudio: Los manglares de los esteros Iguanita $\left(10^{\circ} 37^{\prime} 50^{\prime \prime} \mathrm{N}-85^{\circ} 37^{\prime} 40^{\prime \prime} \mathrm{W}\right)$ y Playa Panamá $\left(10^{\circ} 35^{\prime} 16^{\prime \prime} \mathrm{N}-85^{\circ} 39^{\prime} 26^{\prime \prime} \mathrm{W}\right)$ se encuentran ubicados en la zona de Bahía Culebra, Pacífico Norte de Costa Rica (Fig. 1 y 2). Ambos manglares y las características de la zona de estudio fueron descritos por SamperVillarreal et al. (2012).

Cobertura de los bosques de manglar: Para estudiar los cambios en la cobertura de manglar, se utilizaron fotografías aéreas pancromáticas y a color; e imágenes satelitales de 1945 al 2010 (Cuadro 1 y Cuadro 2). Ambas
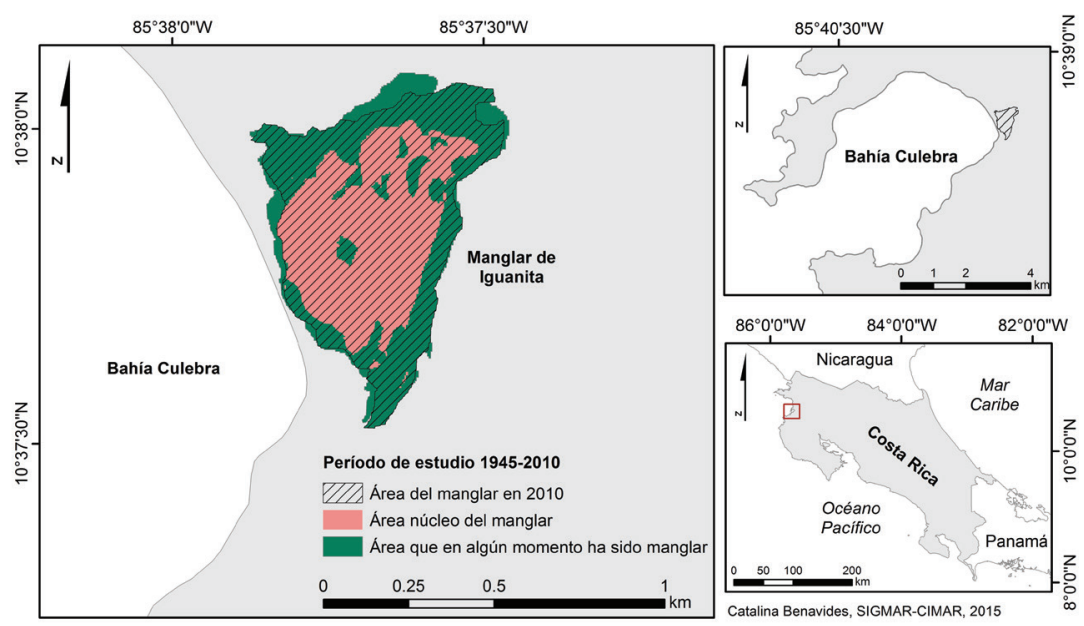

Fig. 1. Variación espacio-temporal del área de manglar de Iguanita, Bahía Culebra, Guanacaste, durante un período de 65 años (1945-2010).

Fig. 1. Spatial and temporal variation of the mangrove forest area of Iguanita, Bahía Culebra, Guanacaste, during a 65-year period (1945-2010). 


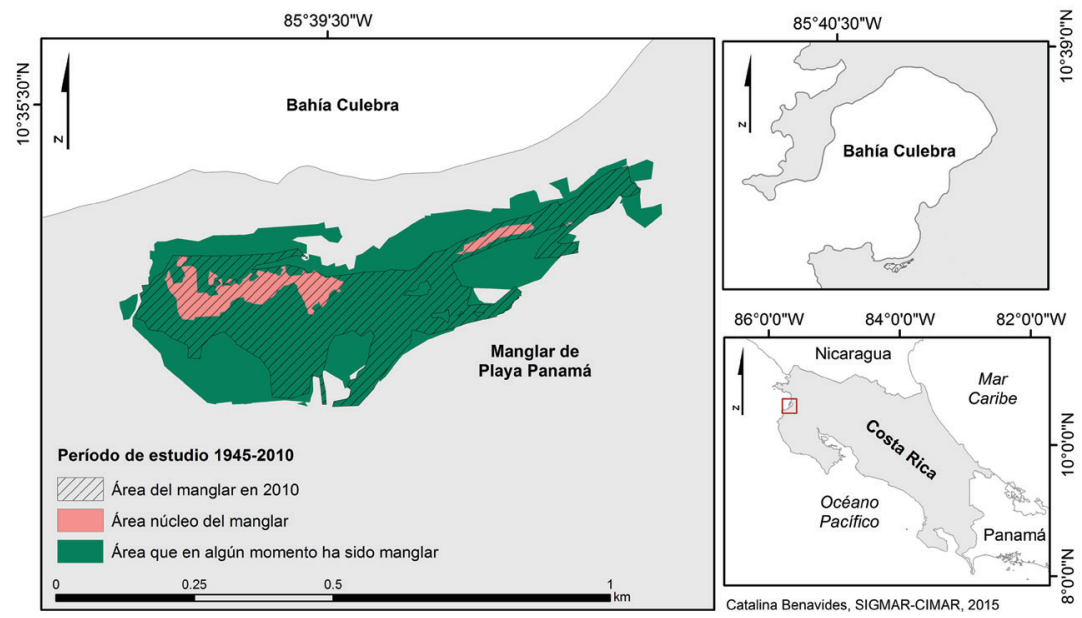

Fig. 2. Variación espacio-temporal del área de manglar de Playa Panamá, Bahía Culebra, Guanacaste, durante un período de 65 años (1945-2010).

Fig. 2. Spatial and temporal variation of the mangrove forest area of Playa Panamá, Bahía Culebra, Guanacaste, during a 65-year period (1945-2010).

CUADRO 1 / TABLE 1

Información de las fotografías aéreas e imágenes utilizadas / Information of the aerial photographs and images used

\begin{tabular}{|c|c|c|c|c|c|c|}
\hline Fecha & Tipo & Escala & Proyecto & Fuente & Manglar Iguanita & Manglar Playa Panamá \\
\hline $01 / 03 / 1945$ & Pancromática & $1: 40000$ & CAW M-1083 & IGNCR & $\mathrm{X}$ & $\mathrm{X}$ \\
\hline $02 / 12 / 1964$ & Pancromática & $1: 10000$ & Hard-Catastro & IGNCR & $\mathrm{X}$ & \\
\hline 24/08/1971 & Pancromática & $1: 20000$ & Nicoya & IGNCR & $\mathrm{X}$ & \\
\hline 24/11/1971 & Pancromática & $1: 20000$ & Nicoya & IGNCR & & $\mathrm{X}$ \\
\hline 21/12/1977 & Pancromática & $1: 20000$ & Valle Tempisque & IGNCR & & $\mathrm{X}$ \\
\hline 05/03/1980 & Pancromática & $1: 10000$ & Bahía Culebra & IGNCR & $\mathrm{X}$ & $\mathrm{X}$ \\
\hline $11 / 12 / 1981$ & Pancromática & $1: 20000$ & INS Guanacaste & IGNCR & & $\mathrm{X}$ \\
\hline 22/07/1987 & Pancromática & $1: 35000$ & Stamp & IGNCR & $\mathrm{X}$ & \\
\hline 03/12/1997 & Color & $1: 40000$ & Terra & IGNCR & $\mathrm{X}$ & $\mathrm{X}$ \\
\hline $10 / 03 / 2003$ & Infrarojo & $1: 40000$ & CARTA 2003 & VI-UCR & $\mathrm{X}$ & $\mathrm{X}$ \\
\hline $04 / 03 / 2005$ & Infrarojo & $1: 40000$ & CARTA 2005 & VI-UCR & $X$ & $\mathrm{X}$ \\
\hline
\end{tabular}

Fuente: IGNCR: Instituto Geográfico Nacional de Costa Rica, VI-UCR: Vicerrectoría de Investigación de la Universidad de Costa Rica.

se obtuvieron en formato digital con una resolución de 1200 dpi. La orto-imagen QuickBird fue descargada de Google Maps, utilizando la extensión Screengrab (Andy, 2007), según la metodología descrita por Fallas (2009). Se hicieron observaciones de campo en 2007 y 2008 (Samper-Villarreal et al., 2012).

Las fotografías fueron corregidas geométricamente usando puntos de control tomados en el campo y el sistema de coordenadas Costa Rica Transversal de Mercator, Datum
WGS84 (CRTM05). Para la georreferenciación se usó una transformación polinomial de primer orden, con un algoritmo de re-muestreo del vecino más cercano, un tamaño de celda de $1 \mathrm{~m}$ y se determinó el error medio cuadrático (RMS), que en promedio fue de 11.3 para Iguanita y 5.6 para Panamá. Esto se hizo usando el programa ArcGIS 10.0 (ESRI, 2012).

Para las imágenes satelitales se hizo la corrección radiométrica, geométrica y atmosférica, usando el programa ENVI 5 (Exelis 
CUADRO 2 / TABLE 2

Características de las imágenes satelitales utilizadas / Characteristics of the satellite images used

\begin{tabular}{|c|c|c|c|c|c|}
\hline Fecha & $\begin{array}{l}\text { Tipo de imagen y } \\
\text { resolución }(\mathrm{m})\end{array}$ & $\begin{array}{l}\text { Resolución espectral Banda: } \\
\text { espectro de banda }\end{array}$ & Fuente & $\begin{array}{l}\text { Manglar } \\
\text { Iguanita }\end{array}$ & $\begin{array}{l}\text { Manglar Playa } \\
\text { Panamá }\end{array}$ \\
\hline $21 / 03 / 2009$ & Rapid Eye (6.5 m) & $\begin{array}{l}\text { 1: } 0.44-0.51 \mu \mathrm{m} \text { (Azul) } \\
\text { 2: } 0.52-0.59 \mu \mathrm{m} \text { (Verde) } \\
\text { 3: } 0.63-0.69 \mu \mathrm{m} \text { (Rojo) } \\
\text { 4: } 0.69-0.73 \mu \mathrm{m} \text { (Infrarrojo) } \\
\text { 5: } 0.76-0.85 \mu \mathrm{m} \text { (Infrarrojo cercano) }\end{array}$ & GIZ1'-PRIAS ${ }^{2}$ & & $\mathrm{X}$ \\
\hline 08/12/2010 & ASTER $(15 \mathrm{~m})$ & $\begin{array}{l}\text { 1: } 0.52-0.60 \mu \mathrm{m} \text { (Verde) } \\
\text { 2: } 0.63-0.69 \mu \mathrm{m} \text { (Rojo) } \\
\text { 3: } 0.78-0.86 \mu \mathrm{m} \\
\text { (Infrarrojo cercano) }\end{array}$ & $\begin{array}{l}\text { PRIAS- } \\
\text { CATHALAC }^{3}\end{array}$ & $\mathrm{X}$ & \\
\hline $10 / 03 / 2010$ & $\begin{array}{l}\text { Quick Bird }(2.4 \mathrm{~m}) \\
\text { Nivel } 17\end{array}$ & $\begin{array}{l}1: 0.44-0.51 \mu \mathrm{m} \text { (Azul) } \\
2: 0.52-0.59 \mu \mathrm{m} \text { (Verde) } \\
\text { 3: } 0.63-0.69 \mu \mathrm{m} \text { (Rojo) }\end{array}$ & $\begin{array}{l}\text { Digital Globe } \\
\text { Google Maps }\end{array}$ & & $\mathrm{X}$ \\
\hline
\end{tabular}

1. GIZ: Agencia de Cooperación Alemana. 2. PRIAS: Laboratorio del Programa de Investigaciones Aerotransportadas. 3. CATHAlAC: Centro del Agua del Trópico Húmedo para América Latina y el Caribe, Panamá. 4. http://archive. digitalglobe.com/archive/showBrowse.php?catID=1010010004F41C05.

VIS, 2012). La imagen RapidEye fue adquirida con un nivel de procesamiento $1 \mathrm{~B}$, que incluye solamente la corrección radiométrica y fue preprocesada según Kalacska y Sánchez-Azofeifa (2008). La orto-rectificación de la imagen se hizo usando un modelo de elevación digital de 90 m, tomado de Shuttle Radar Topography Mission SRTM (Farr et al., 2007). Se usaron las coordenadas y la elevación del punto central de la imagen, para determinar la altura del geoide, usando el sitio web del University NAVSTAR Consortium (UNAVCO, 2013). La corrección atmosférica se hizo con los valores de reflectancia, usando el módulo FLASS del programa ENVI 5.0 (Exelis VIS, 2012).

Para determinar la cobertura de cada tipo de vegetación y sustrato, se hizo una clasificación supervisada con base en los datos de campo. Para esto se usó un índice de vegetación de antocianinas (NDVI) y un modelo de máxima verosimilitud, en el programa ENVI 5.0, según Kalacska y Sánchez-Azofeifa (2008). Sin embargo, dado que los valores de reflectancia para la vegetación de manglar y otros tipos de bosque son similares en la imagen RapidEye, no se obtuvo una separación entre estas coberturas; los índices de separabilidad de bandas según Jeffries-Matusita fueron muy bajos $(<1.0)$ para manglar vs bosque (Richards \& Jia, 1999). Después de hacer varias pruebas, se determinó hacer una interpretación y digitalización de la imagen, con base en los datos de campo.

Para el manglar de Iguanita se obtuvo una imagen ASTER 1A del 2010 (Cuadro 2). A esta imagen se le hizo la calibración radiométrica y la corrección geométrica, usando los valores obtenidos de los metadatos de la imagen y siguiendo el procesamiento descrito por van Ede (2004). La imagen ASTER también fue interpretada visualmente, debido a que la baja resolución espectral y espacial de la imagen no permitió hacer una clasificación supervisada. Para el manglar de Playa Panamá se trabajó con una imagen QuickBird del 2010, incluye la corrección geométrica y radiométrica (sensu Fallas, 2009).

Los límites del área de estudio se determinaron con el borde externo de los manglares y con base en los datos de campo. A partir de ese borde se trazó una zona de amortiguamiento de $200 \mathrm{~m}$ alrededor de cada uno, para estudiar los cambios de cobertura en esas zonas. Después de analizar visualmente las fotografías e imágenes, junto con los datos de campo, se elaboró una lista de los diferentes tipos de cobertura. 
Se hizo la interpretación de las fotografías aéreas e imágenes y la digitalización de las coberturas, incorporando los datos de campo, a una escala de 1:10 000. Se corrigieron los errores de digitalización y se calcularon las áreas y perímetros de cada cobertura, usando el programa ArcGIS 10.0 (ESRI, 2012).

Se trabajó con las capas en formato raster y un tamaño de celda de $4 \mathrm{~m}^{2}$. Con la extensión Spatial Analyst Tools (ESRI, 2012), se generó una capa en la que se pudieran ver los cambios a través del tiempo.

\section{RESULTADOS}

Se determinaron las siguientes categorías de coberturas presentes:

Manglar: Vegetación con especies de mangle como Avicennia germinans, Avicennia bicolor, Rhizophora mangle, Conocarpus erectus y Laguncularia racemosa. En las fotografías aéreas e imágenes, este tipo de cobertura se ve muy homogénea y continua. También se incluyeron zonas con baja densidad de manglar, i.e., con árboles o parches de árboles aislados.

Bosque: Incluye otros tipos de vegetación diferente a manglar, como bosque de galería, bosque seco de transición, bosque seco caducifolio y sabanas arboladas. No se hizo diferencia entre bosque secundario y bosque primario. En las fotografías e imágenes esta cobertura se observa heterogénea.

Sin vegetación: Corresponde a zonas con pastizales, pastizales con árboles o arbustos aislados, suelo descubierto o arena.

Agua: Dulce o salada.

Urbano: Con casas, edificaciones y carreteras.

No determinado: No fue posible determinar el tipo de cobertura, debido a una baja resolución espacial, porque habían nubes o porque la fotografía o imagen no incluía esa porción del sitio.

Manglar de Iguanita: El área total analizada fue de aproximadamente 138 ha. El manglar osciló entre 28.4 ha (1971) y 38.9 ha (2010) (Fig. 3) y 21.3 ha permanecieron espacial y temporalmente como manglar durante los 65 años, esto corresponde al área núcleo de manglar (Fig. 1).

Alrededor del manglar de Iguanita, las categorías con mayor cambio correspondieron a las de bosque y sin vegetación (Fig. 3). El bosque osciló entre 81.7 ha (1945) y 37.1 ha

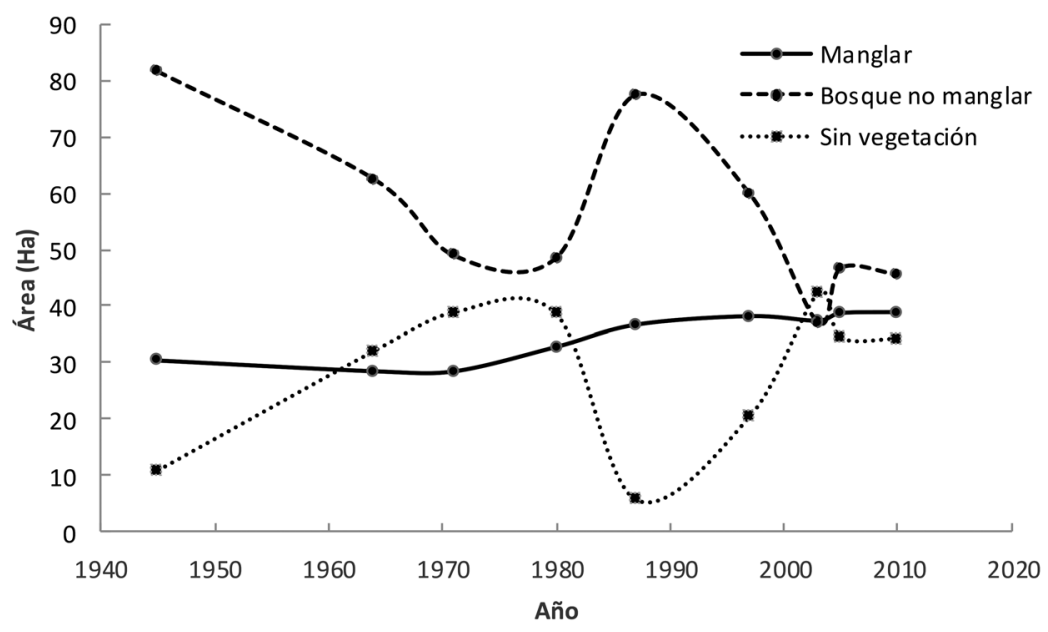

Fig. 3. Cambio de cobertura de vegetación en la zona del manglar de Iguanita entre 1945 y 2010.

Fig. 3. Change in the vegetation cover at Iguanita between 1945 and 2010. 
(2003), mientras que un área de 21.6 ha permaneció invariable como bosque. El área sin vegetación osciló entre 5.6 ha (1987) y 42.2 ha (2003) (Fig. 1). Ésta se correlacionó de manera inversa con el área de manglar y bosque juntas $(\mathrm{r}=-0.98, \mathrm{p}<0.001)$, más que solamente al área de bosque $(\mathrm{r}=-0.92, \mathrm{p}<0.001)$. La tendencia en los alrededores del manglar de Iguanita fue una reducción en la cobertura de bosque en los últimos años, a pesar de que hubo una recuperación en la década de 1990. En 2010 la cobertura del bosque era de 45.6 ha, sólo un $55 \%$ de lo que había en 1945 (Fig. 3).

Manglar de Playa Panamá: El manglar osciló entre 4.8 ha (1977) y 14.8 ha (1997) (Fig. 4). Un área de 1.7 ha fue manglar durante los 65 años (núcleo del manglar, Fig. 2). El manglar de Playa Panamá disminuyó antes de la década de 1980, después de eso hubo una recuperación, incremento y llegó a ocupar un máximo de 14.8 ha en 1997. Después de esto y hasta el 2010, se mantuvo en 12.0 ha (Fig. 4).

Las categorías con mayor cambio alrededor del manglar fueron bosque y sin vegetación. El bosque osciló entre 24.5 ha (1971) y 45.3 ha (1997) (Fig. 4). El área núcleo de bosque fue de 4.5 ha. El área sin vegetación osciló entre 27.7 ha (1980) y 3.7 ha (1997)
(Fig. 4). Ésta se correlaciona de manera inversa con el área conjunta de manglar y bosque $(\mathrm{r}=-0.72, \mathrm{p}=0.019)$. Igual que el manglar, el bosque disminuyó hasta mediados de la década de 1970 y después se recuperó hasta alcanzar un máximo en 1997.

La cobertura urbana alrededor del manglar de Playa Panamá apareció en 1977 y aumentó hasta 2003, cuando alcanzó 4.22 ha. Después de esto se ha mantenido y algunas estructuras o calles han desaparecido.

\section{DISCUSIÓN}

La cobertura de los manglares de Iguanita y de Playa Panamá ha variado desde 1945 hasta 2010; la menor cobertura registrada en el período evaluado fue en la década de 1970 y la mayor en años posteriores. En otras regiones también se ha registrado aumentos en la cobertura de manglar en los últimos años. AlfaroTrejos (2011) informa sobre un aumento en El Salvador, Honduras y Nicaragua, entre 1997 y 2007 de $3.1 \%$. En el Área de Conservación Tempisque en Costa Rica, Cordero-Montoya, Acevedo-Mairena y Calvo-Alvarado (2008) también comunicaron un aumento de $2 \%$ en la cobertura entre 1998 y 2003. Sin embargo, no se tiene claras las razones por las que

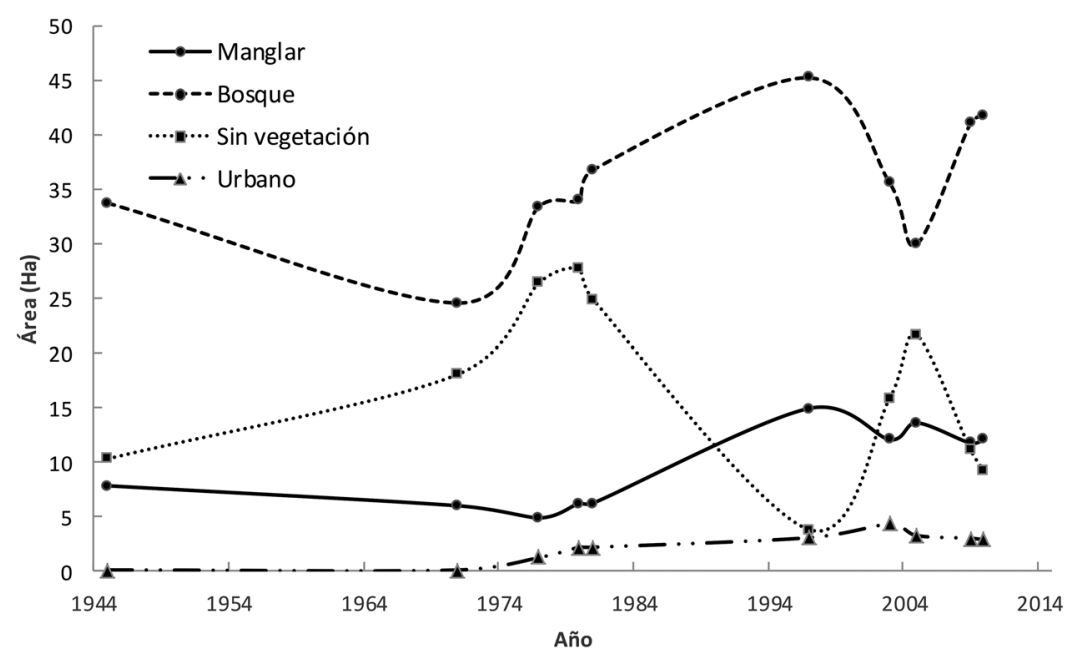

Fig. 4. Cambio de cobertura de vegetación en la zona del manglar de Playa Panamá, entre 1945 y 2010.

Fig. 4. Change in the vegetation cover at Playa Panamá mangrove between 1945 and 2010. 
aumentaron en extensión. Algunos estudios demuestran que la recuperación del manglar puede ser rápida, si se dan las condiciones óptimas para esto (Ross et al., 2006; Alongi, 2008). En menos de 10 años puede lograr un estado de sucesión pionera y en menos de 20 años alcanza un estado maduro joven, caracterizando a los manglares como uno de los ecosistemas más dinámicos (Fromard et al., 1998). Estas condiciones se pueden favorecer por cambios en el nivel del mar o en la geomorfología de la costa, levantamiento o hundimiento de la costa, aumento de precipitación o del volumen de agua que se acumula en el estero, cambios en el uso del suelo en los alrededores y en las políticas de conservación (Jiménez, 1994; Fromard et al., 1998; Flores-Mejía et al., 2010; Lizano, 2013). Aunque no hay datos de este tipo para los manglares estudiados, se sabe que la costa del Pacífico Norte de Costa Rica es muy dinámica. Lizano (2013) ha documentado procesos de erosión en playas de esa región; sin embargo, anota que la erosión podría ser leve en playas que se encuentran en bahías y podría darse por aumento en el nivel del mar, mayor energía del oleaje, hundimientos por subducción de la Placa de Coco bajo la Placa Caribe, efectos del fenómeno de El Niño, mareas astronómicas extraordinarias y cambios en las fuentes y sumideros de los sedimentos desde el océano y el continente. La mayoría de estos fenómenos ocurren en el Pacífico de Costa Rica y podrían afectar el crecimiento de los manglares. A pesar de la evidencia de estos procesos de erosión, otro estudio reciente revela que en la costa del Pacífico más bien hubo un levantamiento de casi $1 \mathrm{~mm} /$ año entre 1992 y 2010 (Biomarcc-SINAC-GIZ, 2013). Por otra parte, los manglares analizados también han sido sujetos de políticas de conservación: el de Iguanita está protegido desde 1994, cuando se creó el Refugio Nacional de Vida Silvestre Iguanita y ambos manglares están protegidos por la Convención RAMSAR, ratificada por ley en 1991.

El aumento en la cobertura de manglar no es una tendencia global. Valiela, Bowen y York (2001) hicieron una revisión de 29 estudios sobre el cambio mundial en su cobertura, y el patrón general fue una disminución de un $35 \%$ entre las décadas de 1980 y 1990. El continente americano registra la mayor disminución de cobertura. Usando imágenes del 2000, Giri et al. (2011) observaron una disminución a nivel global. En Costa Rica varios manglares han sido afectados por tala ilegal y por expansión urbana ligada a infraestructura para actividades turísticas. En agosto 2014 el Tribunal Ambiental denunció la pérdida de 400 hectáreas de manglar en el estero de Puntarenas (Rojas, 2014); ese mismo tribunal condenó a una marina al pago de daños ambientales por la corta de 173 árboles de manglar en Golfito (Redondo, 2008). Asimismo, otros casos de destrucción de manglar en playas de Guanacaste han sido denunciados (Ramírez-Flores, 2009).

Igualmente, ha variado la cobertura boscosa adyacente a los manglares de las zonas estudiadas. Estos resultados son similares a los de otras investigaciones en Centroamérica. Berlanga-Robles, García-Campos, LópezBlanco y Ruiz-Luna (2010) encontraron un patrón similar en los cambios de cobertura en el Pacífico norte de México, entre los años 1973 y 2000. Ellos informaron que las reducciones en cobertura ocurrieron principalmente en el bosque cercano al manglar y no en el manglar (Berlanga-Robles et al., 2010). Alfaro-Trejos (2011) también señala cambios en la cobertura de vegetación alrededor del manglar, que fue afectada por el avance de la frontera agrícola en El Salvador y en Nicaragua. Según dicho estudio, la vegetación arbórea y arbustiva disminuyó en un 9.6 \% entre 1997 y 2007. En el Área de Conservación Tempisque en Costa Rica, Cordero-Montoya et al. (2008) observaron una disminución en la cobertura de bosque de $4.9 \%$ entre 1998 y 2003.

Los cambios en la cobertura de bosque alrededor de los manglares de Iguanita y Playa Panamá concuerdan con otros análisis a un nivel más amplio en Costa Rica. Arroyo-Mora, Sánchez-Azofeifa, Rivard, Calvo, y Janzen (2005) establecen dos períodos de tiempo que marcan la historia del uso del suelo alrededor de Bahía Culebra. El primer período entre 1960 
y 1980, caracterizado por un aumento de las prácticas de ganadería extensiva e intensiva, que aumentaron las tasa de deforestación. El segundo, entre 1980 y 2000, caracterizado por el abandono de esas prácticas y un aumento de la cobertura de bosque secundario (SánchezAzofeifa, Harris, \& Skole, 2001; Joyce, 2006; Calvo-Alvarado, McLennan, Sánchez-Azofeifa, \& Garvin, 2009). Ambos períodos son claramente distinguibles en nuestros resultados con los mismos patrones. Calvo-Alvarado et al. (2009) mencionan que esta recuperación del bosque se da por múltiples factores socioeconómicos, pero resaltan la caída internacional del precio de la carne y el desarrollo de políticas de conservación más efectivas. Después del 2000 no hay mucha información sobre cómo han sido los cambios en la cobertura de bosque en la región; sin embargo, la información disponible parece indicar que la recuperación de bosque secundario se detuvo y que más bien muchos de los bosques recuperados en las décadas de 1980 y 1990 se han perdido, tal y como ocurrió en los alrededores de los manglares de Iguanita y Playa Panamá. Esta pérdida de cobertura de bosque, ha estado ligada al desarrollo inmobiliario, producto del incremento de la actividad turística (Vargas, 2010). En los alrededores del manglar de Playa Panamá, hubo un aumento del uso urbano a partir del 2003, asociado a una pérdida de cobertura de bosque.

El manglar de Playa Panamá actualmente está rodeado por una carretera y algunas obras de infraestructura hacia los sectores sur y oeste, además de la construcción de un proyecto residencial (obs. pers.). Ante un eventual aumento en el nivel del mar, el manglar no podría extenderse tierra adentro. De igual manera, el manglar de Iguanita tiene una topografía que no permitiría su expansión tierra adentro (Samper-Villarreal et al., 2012).

Este y otros estudios señalan que los manglares son ecosistemas muy dinámicos, y para asegurar su adecuada protección, es necesario evaluar también el uso del suelo y la topografía de las áreas aledañas. Es conveniente que la protección de los manglares también incluya la protección de áreas de amortiguamiento a su alrededor, que permita reducir impactos futuros.

La evaluación de cambios espacio-temporales en la cobertura, no necesariamente requiere de inversiones costosas de dinero, las fotografías aéreas e imágenes satelitales, pueden constituir recursos de libre acceso y la única base retrospectiva para comparar con datos de cobertura actuales.

\section{AGRADECIMIENTOS}

Este proyecto fue financiado por la Vicerrectoría de Investigación de la Universidad de Costa Rica, el Laboratorio del Programa de Investigaciones Aerotransportadas (Laboratorio PRIAS-CENAT), el Ministerio de Ambiente Alemán a través de la Agencia de Cooperación Alemana para el Desarrollo (GIZ) y el Centro del Agua del Trópico Húmedo para América Latina y el Caribe (CATHALAC).

\section{RESUMEN}

Los manglares tienen gran importancia ecológica, económica, riqueza natural y prestan servicios ambientales. No obstante, son amenazados por la sobreexplotación, la contaminación y el cambio de uso de suelo. Costa Rica tiene manglares en las costas pacífica y caribeña. Según algunos estudios, la cobertura de manglar ha ido disminuyendo desde la década de 1980. Debido a que estos datos no son actualizados y se basan en estimaciones poco precisas, es necesario hacer una valoración de la extensión actual y la variación de la cobertura en los últimos años, que permita determinar cambios. En esta investigación se estudió la cobertura de dos manglares ubicados en Bahía Culebra, Pacífico Norte: Iguanita y Playa Panamá. Se usaron fotografías e imágenes de satélite para un período de 65 años (1945-2010). Se encontraron cambios espaciotemporales en la cobertura de manglar, bosques adyacentes y áreas sin vegetación. Las menores coberturas de manglar se registraron en la década de 1970, pero aumentaron en años posteriores. Los cambios en la cobertura de bosque alrededor de los manglares de Iguanita y Playa Panamá concuerdan con otros análisis históricos sobre el uso del suelo alrededor de Bahía Culebra. Antes de 1980 se dio un aumento de las prácticas de ganadería extensiva e intensiva, lo que aumentó la tasa de deforestación. Después de 1980 se abandonaron estas prácticas y la cobertura de bosque secundario aumentó hasta el año 2000. Para asegurar una adecuada protección de los manglares, es necesario evaluar 
también las áreas aledañas y establecer zonas de amortiguamiento alrededor, para reducir los impactos futuros.

Palabras clave: ambientes costeros, América Central, cambios, cobertura, fotografías aéreas, imagen satelital, manglares, SIG.

\section{REFERENCIAS}

Alfaro-Trejos, S. (2011). Cobertura y uso de la tierra en el ecosistema de mangle y zona ecotonal del corredor del mangle, desde la Bahía de Jiquilisco, El Salvador hasta el estero Padre Ramos, Nicaragua. Revista Geográfica de América Central, 2(47E), 1-19.

Alongi, D. M. (2008). Mangrove forests: resilience, protection from tsunamis, and responses to global climate change. Estuarine, Coastal and Shelf Science, 76(1), 1-13.

Andy, M. (2007). Screengrab (Version 0.96.3). Extensión de Firefox.

Arroyo-Mora, J. P., Sánchez-Azofeifa, G. A., Rivard, B., Calvo, J. C., \& Janzen, D. H. (2005). Dynamics in landscape structure and composition for the Chorotega region, Costa Rica from 1960 to 2000. Agriculture, Ecosystems \& Environment, 106(1), 27-39.

Berlanga-Robles, C. A., García-Campos, R. R., LópezBlanco, J. \& Ruiz-Luna, A. (2010). Patrones de cambio de coberturas y usos del suelo en la región costa norte de Nayarit (1973-2000). Boletín del Instituto de Geografia, UNAM, 72, 7-22.

Bilio, M., Coze, A. S. \& Portocarrero, A. H. (1999). Los manglares, los camarones y el desarrollo de las áreas costeras en el Istmo Centroamericano. EC Fisheries Cooperation Bulletin, 12, 18-20.

Biomarcc-SINAC-GIZ. (2013). Análisis de vulnerabilidad de las zonas oceánicas y marino-costeras de Costa Rica frente al cambio climático. San José, Costa Rica: Deutsche Gesellschaft für Internationale Zusammenarbeit (GIZ) GmbH.

Calvo-Alvarado, J., McLennan, B., Sánchez-Azofeifa, A., \& Garvin, T. (2009). Deforestation and forest restoration in Guanacaste, Costa Rica: putting conservation policies in context. Forest Ecology and Management, 258(6), 931-940.

CATHALAC. (2012). Centro del Agua del Trópico Húmedo para América Latina y el Caribe. http://www. cathalac.org/en/

Cordero-Montoya, R., Acevedo-Mairena, H., \& CalvoAlvarado, J. C. (2008). Cambio de la cobertura de la tierra para el Área de Conservación Tempisque 1998-2003, Guanacaste, Costa Rica. Kurú: Revista Forestal (Costa Rica), 5(15), 1-15.
Duke, N. C., Meynecke, J.-O., Dittman, S., Ellison, A. M., Anger, K., Berger, U., Cannicci, S., ..., \& DahdoughGuebas, F. (2007). A world without mangroves? Science, 317, 41 .

ESRI. (2012). ArcGIS (Version 10.0). California, E.U.A.: Enviromental System Research Institute.

Ewel, K., Twilley, R., \& Ong, J. (1998). Different kinds of mangrove forests provide different goods and services. Global Ecology \& Biogeography Letters, 7(1), 83-94.

Exelis VIS. (2012). ENVI (Versión 5.0) [Environment for Visualizing Images]. Colorado, E.U.A.: Exelis Visual Information Solutions.

Fallas, J. (2009). Descarga de imágenes de alta resolución desde Google Maps, imágenes satelitales de alta resolución a su alcance. Geoambiente, Tecnologías de la Información Geoespacial, Escuela de Ciencias Ambientales, Universidad Nacional. Heredia, Costa Rica.

FAO (2007a). The World's Mangroves 1980-2005 (Informes Nacionales). Roma, Italia: Organización de las Naciones Unidas para la Agricultura y la Alimentación.

FAO (2007b). Mangroves of North and Central America 1980-2005 (Informes Nacionales). Roma, Italia: Organización de las Naciones Unidas para la Agricultura y la Alimentación.

Farr, T.G., Rosen, P. A., Caro, E., Crippen, R., Duren, R., Hensley, S., Kobrick, ..., \& Alsdorf, D. (2007). The Shuttle Radar Topography Mission. Reviews of Geophysics, 45(2), p.RG2004.

Flores-Mejía, M. A., Aguirre-Vallejo, A., Flores-Hernández, M., \& Guarddo-Govea, X. (2010). El impacto que produce el sector turismo en los manglares de las costas mexicanas. Contactos, 77, 33-38.

Fromard, F., Puig, H., Mougin, E., Marty, G., Betoulle, J. L., \& Cadamuro, L. (1998). Structure, above-ground biomass and dynamics of mangrove ecosystems: new data from French Guiana. Oecologia, 115(1-2), 39-53.

Giri, C., Ochieng, E., Tieszen, L. L., Zhu, Z., Singh, A., Loveland, T., \& Duke, N. (2011). Status and distribution of mangrove forests of the world using earth observation satellite data. Global Ecology and Biogeography, 20(1), 154-159.

Jiménez, J. A. (1994). Los manglares del Pacifico Centroamericano. Heredia, Costa Rica: EFUNA, Universidad Nacional.

Jiménez, J. A. \& Soto, R. (1985). Patrones regionales en la estructura y composición florística de los manglares de la Costa Pacífica de Costa Rica. Revista Biología Tropical, 33, 25-37. 
Joyce, A. T. (2006). Land Use Change in Costa Rica: 1966 2006, as Influenced by Social, Economic, Political, and Enviromental Factors. San José, Costa Rica: Litografía e Imprenta LIL S.A.

Kalacska, M., \& Sánchez-Azofeifa, G. A. (2008). Hyperspectral Remote Sensing of Tropical and Subtropical Forest. Boca Raton, Florida: CRC Press, United States of America.

Lizano, O. G. (2013). Erosión en las playas de Costa Rica, incluyendo la Isla del Coco. InterSedes, 14(27), 06-27.

Loría-Naranjo, M., Samper-Villarreal, J., \& Cortés, J. (2014). Potrero Grande and Santa Elena mangrove forest structure, Santa Rosa National Park, North Pacific, Costa Rica. Revista de Biología Tropical, 62(Suppl. 4), 33-41.

MIRENEM. (1993, Junio). Informe de Costa Rica. Taller sobre manejo de humedales y manglares en Centro América y el Caribe, Miami, Florida, EEUU.

Morales-Cabalceta, T. G. (1983). Evaluación de la extracción de corteza de mangle, Guanacaste-Costa Rica (Informe Práctica de Especialidad, Bach. Ingeniería Forestal). Instituto Tecnológico de Costa Rica, Cartago, Costa Rica.

Pizarro, F. \& Angulo, H. (1993). Diagnóstico de los manglares de la costa Pacífica de Costa Rica. Heredia, Costa Rica: CATIE, UNA.

Polidoro, B. A., Carpenter, K. E., Collins, L., Duke, N. C., Ellison, A. M., Ellison, J. C., Farnsworth, E. J., ..., \& Yong, J. W. H. (2010). The loss of species: mangrove extinction risk and geographic areas of global concern. PLoS One, 5(4), e10095.

Ramírez-Flores, E. (2009). Recurso de amparo contra varias instituciones: Investigan destrucción de manglar en hotel Riu. Semanario Universidad. Retrieved from: http://www.semanariouniversidad.ucr.cr/ component/content/article/537-Pa\%C3\%ADs/1730recurso-de-amparo-contra-varias-instituciones-investigan-destruccion-de-manglar-en-hotel-riu-.html

Redondo, A. (2008). Desarrollo turístico descontrolado. Ambientico, 181, 7-10.

Richards, J. A. \& Jia, X. (1999). Remote Sensing Digital Image Analysis-An Introduction (3 ${ }^{\text {rd }}$ ed.). Berlín, Alemania: Springer Verlag.

Rojas, P. (2014, Agosto 26). Tribunal Ambiental denuncia pérdida de 400 hectáreas del manglar de Puntarenas, casos irán a Fiscalía. CRHoy.com. Retrieved from: http://www.crhoy.com/tribunal-ambiental-denunciaperdida-de-400-hectareas-del-manglar-de-puntarenas-casos-iran-a-fiscalia/
Ross, M. S., Ruiz, P. L., Sah, J. P., Reed, D. L., Walters, J., \& Meeder, J. F. (2006). Early post-hurricane stand development in fringe mangrove forests of contrasting productivity. Plant Ecology, 185(2), 283-297.

Samper-Villarreal, J., Cortés, J., \& Benavides-Varela, C. (2012). Description of the Panamá and Iguanita mangrove stands of Bahía Culebra, North Pacific coast of Costa Rica. Revista de Biología Tropical, 60(Suppl. 2), 109-120.

Sánchez-Azofeifa, G. A., Harriss, R. C., \& Skole, D. L. (2001). Deforestation in Costa Rica: A quantitative analysis using remote sensing imagery. Biotropica, 33(3), 378-384.

Soto, R. (1988). Deforestación y otros tipos de perturbaciones en los manglares del Pacífico de Costa Rica. In INIREB (Ed.), Memorias: Ecología y conservación del delta de los ríos Usumacinta y Grijalva (pp. 177200). México: Instituto Nacional de Investigación sobre Recursos Bióticos, División Tabasco y Gobierno del Estado de Tabasco.

Soto, R. \& Jiménez, J. A. (1982). Análisis fisonómico estructural del manglar de Puerto Soley, La Cruz, Guanacaste, Costa Rica. Revista de Biología Tropical, 30(2), 161-168.

UNAVCO. (2013). University NAVSTAR Consortium https://www.unavco.org/

Valiela, I., Bowen, J. L., \& York, J. K. (2001). Mangrove forests: One of the world's threatened major tropical environments. Bioscience, 51(10), 807-815.

van Ede, R. (2004). Destriping and Geometric Correction of an ASTER Level 1A Image. Netherlands: Department of Physical Geography, Faculty of GeoSciences, Ultrecht University.

Vargas, E. (2010). Impactos ambientales de los desarrollos turísticos y residenciales: Identificación y análisis de impactos ambientales relevantes de los desarrollos turísticos y residenciales en la costa pacífica de Costa Rica. San José, Costa Rica: Center for Responsible Travel, Standford University.

Zamora-Trejos, P. (2006). Manglares. In V. Nielsen-Muñoz \& M. A. Quesada-Alpízar (Eds.), Informe Técnico: Ambientes marinos costeros de Costa Rica (pp. 23-39). San José, Costa Rica: Comisión Interdisciplinaria Marino Costera de la Zona Económica Exclusiva de Costa Rica. CIMAR-CI.

Zamora-Trejos, P. \& Cortés, J. (2009). Los manglares de Costa Rica: el Pacífico norte. Revista de Biología Tropical, 57(3), 473-488. 